<smiles>[C]1C=C[CH-]C1</smiles>

\title{
Características de la crianza democrática implementadas por padres bogotanos
}

\author{
Characteristics of democratic parenting implemented by Bogota parents \\ Características da parentalidade democrática implementada por pais de Bogotá
}

\author{
Adriana Vargas \\ Corporación Universitaria Iberoamericana (Colombia) \\ Diana Duarte \\ Corporación Universitaria Iberoamericana (Colombia) \\ Diana Ariza \\ Corporación Universitaria Iberoamericana (Colombia) \\ D0l: https://doi.org/10.35622/j.rep.2021.04.006
}

Recibido 08/06/2021/ Aceptado 16/11/2021

RESUMEN. El estilo de crianza democrático se ha posicionado en los últimos años, a raíz de los beneficios que demuestra tener en los niños y adolescentes, no obstante, se ha evidenciado el desconocimiento de los padres, sobre los beneficios y características que lo componen. Con el objetivo de describir las características de la crianza democrática que implementan con mayor regularidad un grupo de padres bogotanos se utilizó una investigación cuantitativa de tipo descriptiva, con una muestra no representativa de 100 padres residentes en Bogotá. Se identificó que los padres implementan con mayor regularidad 3 características: buscar el desarrollo de la autonomía de sus hijos, comunicarse de manera clara con ellos e interesarse por su formación y educación. Muchos padres no emplean la razón como forma de corrección, datos que coinciden con las estadísticas del Observatorio de Salud de Bogotá. Se concluye que la implementación de los estilos de crianza influirá en el desarrollo de los niños y adolescentes, por esto, se considera importante promover el estilo de crianza democrático, al interior del núcleo familiar.

PALABRAS CLAVE: Familia, estilos de crianza, crianza democrática, padres democráticos.

\begin{abstract}
The democratic parenting style has been positioned in recent years due to the benefits it proves to have in children and adolescents; however, the ignorance of parents about the benefits and characteristics that compose it has been evidenced. Therefore, descriptive quantitative research was used to describe the aspects of democratic upbringing that a group of Bogota parents implements with greater regularity, with a non-representative sample of 100 parents residing in Bogota. It was identified that parents implement three characteristics more regularly: seek the development of their children's autonomy, communicate clearly with them and take an interest in their training and education. Furthermore, many parents do not use reason as a form of correction, data that coincide with the statistics of the Bogotá Health Observatory. It is concluded that the implementation of parenting styles will influence the development of children and adolescents. Therefore, it is considered essential to promote the democratic parenting style within the family nucleus.
\end{abstract}

KEYWORDS: Family, parenting styles, democratic parenting, democratic parents. 
RESUMO. 0 estilo parental democrático tem se posicionado nos últimos anos, em decorrência dos benefícios que prova ter nas crianças e adolescentes, porém, fica evidenciado o desconhecimento dos pais sobre os benefícios e características que o compõem. Para descrever as características da educação democrática que um grupo de pais de Bogotá implementa com maior regularidade, foi utilizada uma pesquisa quantitativa descritiva, com uma amostra não representativa de 100 pais residentes em Bogotá. Identificou-se que os pais implementam 3 características com mais regularidade: buscam 0 desenvolvimento da autonomia dos filhos, comunicam-se com clareza e se interessam pela sua formação e educação. Muitos pais não usam a razão como forma de correção, dados que coincidem com as estatísticas do Observatório de Saúde de Bogotá. Conclui-se que a implantação de estilos parentais influenciará no desenvolvimento de crianças e adolescentes, portanto, considera-se importante promover o estilo parental democrático, no núcleo familiar.

PALABRAS CLAVE: Família, estilos parentais, paternidade democrática, pais democráticos.

\section{INTRODUCCIÓN}

La familia es la primera unidad, con la que se establece contacto social, se empiezan a desarrollar diferentes habilidades y se establecen patrones de conducta que están guiados por los estilos de crianza que emplean los principales referentes de esta unidad, es decir, los padres (Infante \& Martines, 2016). La familia es uno de los microsistemas más influyentes en cuanto a la adquisición de la personalidad y de los roles, que posteriormente serán puestos en práctica en contexto (Parra \& Rubio, 2017).

A raíz de la industrialización y la digitalización se han ido presentando transformaciones culturales con el pasar del tiempo, que han afectado todos los sistemas, y la familia no ha sido la excepción. De esta manera, se gestaron cambios al interior de esta estructura social, impulsando la igualdad de género, la incorporación de la mujer al mercado laboral y la implementación de nuevos estilos de crianza, causado por la necesidad de adaptación al cambio (Infante \& Martínez, 2016). No obstante, Climen (2006) citado por Moreno (2013) reconoce que estos mismos cambios han generado que la educación sea cada vez más compleja, y los padres aun no logren establecer patrones de crianza adecuados que puedan prevalecer en el tiempo.

Como lo expone el observatorio de salud de Bogotá, en el año 2019, se presentaron cifras alarmantes sobre los casos de maltrato (21.223), negligencia parental (12.229) y maltrato físico (3.386), contra niños, niñas y adolescentes. Situación que demuestra la necesidad de promover un estilo de crianza alternativo que fomente el respeto, la unión y la comunicación como forma principal para abordar las problemáticas que se viven dentro del microsistema familiar. 
En este sentido, se hace necesario reconocer y entender el rol de los padres, en la familia, en la sociedad, y en la transmisión de pautas de crianza. Márquez y Gaeta (2017) señalan que los padres influyen de manera directa e indirecta en el desarrollo de las competencias emocionales, sociales, en la educación y en el fortalecimiento del entorno familiar. A partir de las creencias que éstos adoptan, guían y orientan la crianza, asumiendo que su forma de criar a los hijos es la más adecuada a partir de las necesidades particulares que cada familia experimenta. (Solís et al., 2007; citado en Infante \& Martínez, 2016).

Los estilos, pautas y modelos de crianza, deben ser entendidos y asumidos como conceptos similares que hacen referencia al mismo termino. Los cuales se relacionan directamente con las formas, creencias y actitudes que ponen en práctica los padres, con relación a la salud, la nutrición, la educación, la interacción social y el ambiente físico, en la crianza de sus hijos y que influyen directamente en los niños, niñas y adolescentes (Ramírez, 2005, citado por Jorge \& González; 2017). Estos estilos, reciben influencia de la cultura y de las tradiciones sociales que se viven en determinada época, o que han sido transmitidas generación a generación (Arciniegas, et al 2018).

Diana Baumrind (1966) es una de las principales exponentes con relación a los estilos de crianza, ella menciona los efectos que estos tienen sobre el desarrollo del infante y adolescente. Además, propone tres principales estilos, los cuales son, el autoritario, el permisivo y el democrático (citado en Guallpa \& Loja 2015).

El estilo autoritario, se caracteriza por el control y el establecimiento de reglas rígidas que exigen al niño o adolescente asumir una postura inflexible de madurez. En este estilo, la comunicación activa y la expresión de afecto no predominan, por lo cual, no se crean muchos lazos afectivos de calidad (Ramírez, 2005 citado en Castillo 2016). Los padres presentan múltiples restricciones de comportamiento, valoran y exigen la disciplina, no fomentan en muchas ocasiones la autonomía de los hijos y ejercen conductas de subordinación hacia ellos, disminuyendo la seguridad en sí mismos y causando dificultades en la adaptación social del niño y el adolescente (Torio et al., 2008, citado en Capano et al., 2016).

Se ha evidenciado también, que los niños cuyos padres ejercen prácticas autoritarias, pueden presentar problemas o dificultades a nivel emocional, debido al poco o nulo apoyo 
que reciben de sus padres (McLeod y Chaffee, 1972, citado en Rojas, 2015). La mayoría estos niños, son tímidos y retraídos con sus pares, ya que no recibieron tanta estimulación, para enfrentarse al contexto social y establecer relaciones positivas, se pueden irritar con mucha facilidad y no ser tan alegres o extrovertidos (Maccoby y Martin 1983, citado en Capano et al., 2016). Estos niños también pueden presentar problemas de desconfía, alta ansiedad y poca creatividad (Castillo, 2016).

La Rosa (1986) citado en Girardi y Velasco (2006) menciona que los padres son más autoritarios que las madres, y que aquellos padres que tienen entre 17 a 20 años, ejercen patrones de crianza más autoritarios.

El estilo permisivo, se caracteriza por el poco establecimiento de normas y reglas, por la falta de fijación de límites claros y la poca o nula imposición de orden (Castillo, 2016). Los padres que implementan este estilo de crianza son muy condescendientes con sus hijos y presentan altos grados de afectividad, lo cual causa y permite que el infante o adolescente realice cualquier actividad o actúe como él quiere (Acosta et al., 2015).

De esta manera, los niños que tiene padres permisivos son alegres y espontáneos, pero pueden presentar conductas inapropiadas o antisociales. Al no tener límites de comportamiento claros, desconocen en muchas ocasiones el respeto que deben tener con y hacia las demás personas, pueden también ser agresivos e impulsivos y tener poca confianza en sí mismos (Castillo, 2016).

El estilo democrático, se caracteriza por propiciar el diálogo entre padres e hijos, promover su independencia, establecer normas claras de comportamiento y establecer expectativas reales que cada miembro de la familia debe cumplir (Baumrind, 1991, citado en Rojas 2015).

Los padres que implementan este estilo de crianza tienen confianza en ellos como padres, emplean una comunicación clara y buscan fortalecer la empatía en sus hijos. De esta manera ellos tendrán en cuenta las necesidades de los demás y serán niños o adolescentes consientes y respetuosos con el otro, escuchan, toman en cuenta la opinión y sentimientos de sus hijos frente a las situaciones familiares, pero son los padres quienes toman la última decisión (Acosta, Toro \& Guerrero 2015).

Baumrind (1971, citado en Castillo (2016) afirma que este estilo, favorece la independencia del niño y la resolución de conflictos a partir de la comunión activa, se promueve la 
interacción social positiva y la cooperación. La sensibilidad de estos padres es mayor, ya que escuchan y atienden las necesidades de sus hijos, también los animan a ser autónomos, respetuosos e independientes (Guallpa y Loja. 2015).

En cuanto a los hijos de padres democráticos, se puede resaltar que tienen una mejor expresión y control emocional. También presentan un mejor rendimiento académico, más autoestima, son capaces de establecer y cumplir metas realistas y positivas. En cuanto a la relación con sus pares, presentan conductas menos agresivas y más tolerantes (Capano et al., 2016). Cabrera et al., (2006) citado de Moreno et al., (2017) mencionan que este estilo, también se caracteriza por el alto y estable nivel de afecto que se presenta al interior de la familia, se brindan refuerzos a los comportamientos positivos, se toman en cuenta las capacidades de los hijos para establecer tareas, y por medio del dialogo, se evita la arbitrariedad y se establecen normas a partir de acuerdos justos.

Teniendo en cuenta diferentes hallazgos investigativos, Guzmán et al., (2019) afirman que la implementación del estilo democrático propicia que los hijos se conviertan en personas competentes, responsables, hábiles a nivel social, con fácil y alto nivel de adaptación, emprendedoras y con mayores posibilidades de asumir nuevos retos. Favorece que los niños comprendan el valor de la empatía, de la justicia y realicen juicios sobre su propio comportamiento.

Se puede establecer que el estilo de crianza democrático, está compuesto por las siguientes características: claridad en la comunicación, fortalecimiento de la empatía, promoción de la autonomía e independencia, establecimiento de normas claras de comportamiento, permitir y fortalecer la expresión y control emocional, tener en cuenta la opinión de los hijos al tomar decisiones, interesarse por la formación y educación de los hijos, comprender y respetar su autonomía, responder a las necesidades físicas, académicas y sociales del infante y considerar importante propiciar la equidad de género. Este estilo de crianza se considera el más apto para educar a los hijos, debido a los beneficios que genera en el desarrollo cognitivo, social, emocional y conductual del infante. (Quiroz et al, 2013) citado por Gutiérrez \& Ardila 2016).

Algunos resultados investigativos amplían la comprensión de estos estilos, en especial del democrático. 
En una investigación realizada por Puyana \& Mosquera (2005), citado en Carrillo, et. al (2016) se pudo establecer que, aunque algunos padres colombianos vivan bajo un paradigma tradicional o hegemónico y asuman el rol de proveedores, reconocen la importancia de la responsabilidad y protección paternal. Asimismo, consideran esencial tener buenas interacciones con sus hijos y expresar emociones positivas que favorezcan la relación.

Por otra parte, García et al., (2017) lograron establecer que, aunque los padres sientan o perciban tener un estilo de crianza democrático, en el contexto escolar se evidencia que algunos padres emplean castigos físicos, no brindan normas claras a sus hijos y no dedican tiempo de calidad para acompañarlos y orientarlos en las actividades académicas. Esto demuestra que implementan pocas o nulas características de este estilo de crianza.

Con relación a este fenómeno, se presentó ante la cámara de representantes de Colombia, una ley que busca prohibir el castigo físico como forma de educar a los niños, niñas y adolescentes, promoviendo en este proyecto, que el estado garantice y brinde a los padres de familia, herramientas para que las pautas de crianza sean más respetuosas, positivas y basadas en el diálogo activo, buscando transformar los microsistemas familiares (Colprensa, 2020). Por lo anterior, el objetivo de la presente investigación es describir las características de la crianza democrática implementadas con mayor regularidad por un grupo de padres Bogotanos.

\section{MATERIAL Y MÉTODOS}

\section{Diseño}

Se realizó una investigación cuantitativa de tipo descriptiva, para establecer los patrones de comportamiento de los padres bogotanos, en cuanto a la implementación de las características de la crianza democrática (Hernández et al., 2014)

\section{Participantes}

La población objeto de esta investigación, son los padres que actualmente residen en Bogotá. 
La muestra estuvo compuesta por 100 padres bogotanos. La selección se realizó mediante el tipo de muestro no probabilístico por conveniencia, la cual permite seleccionar a los participantes que se tienen disponibles (Hernández et al., 2014).

\section{Instrumento}

Encuesta: permite describir las características de la población, mediante preguntas ya establecidas, facilita el análisis de aspectos o dimensiones de la población objeto, una de las ventajas es que la información es recolectada de manera estándar, lo que permite medir el fenómeno de estudio y contrastarlo con anteriores investigaciones (Echeverria, 2016).

En esta investigación, se diseñó y aplico una encuesta denominada "Cuestionario de participación para padres residentes en la cuidad de Bogotá", el cual contiene 6 preguntas sociodemográficas (ítem 1-6) y una pregunta general que contextualiza el tema y brinda los datos permitentes para cumplir el objetivo (ítem 7). Se describen 15 características que componen la crianza democrática, los padres de familia solo debían seleccionar aquellas 5 características que implementen con mayor regularidad en la crianza de sus hijos.

\section{Procedimiento}

Se realizo una revisión teórica amplia, para conocer y comprender el fenómeno de estudio. Una vez diseñado el marco de referencia, se procedió a analizar cuál era el mejor instrumento para la investigación.

Fase 1. Diseño del instrumento.

Fase 2. Aplicación, se envió de manera masiva a la población objeto, mediante un formulario de Google, que facilitó el acceso a población más lejana. En este instrumento se aclaró a los padres que su desarrollo era con fines netamente académicos y se solicitó la autorización de tratamiento de datos, para realizar el análisis. La duración aproximada de aplicación de este instrumento fue de 2 a 3 minutos por persona aproximadamente.

Fase 3. Los resultados fueron analizados mediante Excel a través de tablas dinámicas que arrojaron resultados exactos.

Fase 4. Presentación de resultados.

\section{Consideraciones éticas}


La información obtenida por los participantes se analizó y manejo bajo estricta confidencialidad, respeto y protección de los derechos de los participantes, a partir de la resolución 8430 de 1993 del Ministerio de Salud.

\section{RESULTADOS}

A continuación, se presentan a nivel descriptivo los resultados obtenidos en la investigación a través de la aplicación del instrumento "Cuestionario de participación para padres Bogotanos".

En la tabla 1, se observan las características de la crianza democrática implementadas con mayor regularidad por los padres participantes, dentro de ellas se encuentran, considerar importante que los hijos sean independientes y autónomos, comunicarse de manera clara con ellos e interesarse por la formación y educación de los hijos (ver tabla 1).

También se evidencia que las características implementadas con menor frecuencia son; brindar herramientas a los hijos para solucionar problemas, buscar satisfacer la necesidades físicas, emocionales y académicas de los hijos a partir de las capacidades que cada padre posea y basarse en la razón y no en la imposición de la fuerza.

Tabla 1. Características implementadas con mayor y menor regularidad.

Características de la crianza democrática

Porcentaje de

implementación

Considero importante que mis hijos sean independientes y autónomos.

$59 \%$

Me comunico de manera clara con mis hijos.

$55 \%$

Me intereso por la formación y educación de mis hijos.

$52 \%$

Tomo en cuenta la opinión de mis hijos para tomar las decisiones. $\quad$ 44\%

Cuando mis hijos tienen comportamientos positivos lo reconozco y $43 \%$ motivo.

Enseño a mis hijos a expresar sus sentimientos de manera abierta. $\quad$ 40\%

Dialogo con mis hijos al menos una vez al día. 39\%

Apoyar emocionalmente a mis hijos es importante para mí. 35\% 
Considero fundamental desarrollar en mis hijos la empatía.

$24 \%$

Evito el castigo físico para corregir un mal comportamiento.

$20 \%$

Considero que los roles de género deben ser equitativos.

$19 \%$

Entiendo que mi hijo siente y piensa de manera autónoma.

$19 \%$

Brindo herramientas a mis hijos para la resolución de conflictos.

$17 \%$

Desde mis capacidades busco satisfacer las necesidades afectivas, 17\% físicas y académicas de mis hijos.

Me baso en la razón y no en la imposición de la fuerza al criar a mis hijos. 17\%

En la tabla 2, se observan las características implementadas por rangos de edad, los padres del primer rango consideran importante que sus hijos sean independientes y autónomos. Se interesan por su formación y educación con mayor regularidad, pero desde sus capacidades buscan satisfacer las necesidades de sus hijos con poca frecuencia (ver tabla 2).

Los padres del segundo rango, implementan con regularidad la comunicación con sus hijos, consideran importante que sean independientes y autónomos. Se interesan por su formación, por otra parte, no brindan herramientas para solucionar conflictos, no entienden que piensen de manera autónoma, no evitan el castigo físico y no se basan en la razón con regularidad.

En cuanto a los padres del tercer rango, todos implementan la comunicación, y la mayoría apoyan emocionalmente a sus hijos. No obstante, no brindan herramientas para la solución de conflictos, no desarrollan la empatía, no satisfacen las necesidades de sus hijos y no entienden que ellos piensen de manera autónoma con frecuencia.

\section{Tabla 2. Características implementadas por rango de edad}

\begin{tabular}{lllc}
\hline Características de la crianza democrática & 18 a 39 & 40 a 59 & $60>$ \\
\hline Apoyar emocionalmente a mis hijos. & $34 \%$ & $32 \%$ & $75 \%$ \\
Brindar herramientas para la resolución de conflictos. & $18 \%$ & $17 \%$ & \\
Considero fundamental desarrollar la empatía. & $27 \%$ & $22 \%$ & \\
Considero importante que sean independientes y autónomos. & $66 \%$ & $53 \%$ & $25 \%$ \\
Considero que los roles de género deben ser equitativos. & $18 \%$ & $20 \%$ & $25 \%$ \\
Cuando tienen comportamientos positivos los reconozco y motivo. & $45 \%$ & $43 \%$ & $25 \%$ \\
\hline
\end{tabular}




\begin{tabular}{llll}
\hline Desde mis capacidades busco satisfacer las necesidades. & $9 \%$ & $30 \%$ & \\
Dialogo con mis hijos al menos una vez al día. & $36 \%$ & $43 \%$ & $50 \%$ \\
Enseño a mis hijos a expresar sus sentimientos. & $46 \%$ & $33 \%$ & $25 \%$ \\
Entiendo que siente y piensa de manera autónoma. & $21 \%$ & $17 \%$ & \\
Evito el castigo físico para corregir un mal comportamiento. & $21 \%$ & $17 \%$ & $25 \%$ \\
Me baso en la razón y no en la imposición de la fuerza. & $14 \%$ & $17 \%$ & $50 \%$ \\
Me comunico de manera clara con mis hijos. & $46 \%$ & $63 \%$ & $100 \%$ \\
Me intereso por la formación y educación. & $52 \%$ & $53 \%$ & $50 \%$ \\
Tomo en cuenta la opinión de mis hijos para tomar las decisiones. & $46 \%$ & $40 \%$ & $50 \%$ \\
\hline
\end{tabular}

Fuente: elaboración propia

En cuanto al género, se evidencia que los padres identificados con ambos géneros consideran importante que sus hijos sean independientes y autónomos. Se interesan por la formación y educación de los hijos. Por otro lado, los padres de género femenino no brindan herramientas a los hijos para la resolución de conflictos con regularidad, y los padres de género masculino, con poca frecuencia se basan en la razón para educar a sus hijos y desde sus capacidades no buscan satisfacer las necesidades afectivas, físicas y adámicas. (Ver tabla 3).

Tabla 3. Características implementadas por género

\begin{tabular}{|c|c|c|}
\hline Características de la crianza democrática & G femenino & G masculino \\
\hline Apoyar emocionalmente a mis hijos. & $34 \%$ & $41 \%$ \\
\hline Brindar herramientas para la resolución de conflictos. & $18 \%$ & $12 \%$ \\
\hline Considero fundamental desarrollar la empatía. & $22 \%$ & $36 \%$ \\
\hline $\begin{array}{l}\text { Considero importante que sean independientes y } \\
\text { autónomos. }\end{array}$ & $59 \%$ & $59 \%$ \\
\hline Considero que los roles de género deben ser equitativos. & $19 \%$ & $18 \%$ \\
\hline $\begin{array}{l}\text { Cuando tienen comportamientos positivos los reconozco y } \\
\text { motivo. }\end{array}$ & $42 \%$ & $47 \%$ \\
\hline Desde mis capacidades busco satisfacer las necesidades. & $19 \%$ & $5 \%$ \\
\hline Dialogo con mis hijos al menos una vez al día. & $39 \%$ & $41 \%$ \\
\hline Enseño a mis hijos a expresar sus sentimientos. & $39 \%$ & $47 \%$ \\
\hline Entiendo que siente y piensa de manera autónoma. & $21 \%$ & $12 \%$ \\
\hline
\end{tabular}




\begin{tabular}{lll}
\hline Evito el castigo físico para corregir un mal comportamiento. & $19 \%$ & $24 \%$ \\
Me baso en la razón y no en la imposición de la fuerza. & $19 \%$ & $5 \%$ \\
Me comunico de manera clara con mis hijos. & $58 \%$ & $41 \%$ \\
Me intereso por la formación y educación. & $50 \%$ & $65 \%$ \\
Tomo en cuenta la opinión de mis hijos para tomar las & $43 \%$ & $47 \%$ \\
decisiones. &
\end{tabular}

Fuente: elaboración propia

\section{DISCUSIÓN}

El objetivo de esta investigación fue describir las características de la crianza democrática que implementan con mayor regularidad los padres bogotanos. Se encontró que las características más implementadas por los padres son; considerar importante que los hijos sean independientes y autónomos, comunicarse de manera clara con ellos e interesarse por la formación y educación de sus hijos. De acuerdo con Capano et al., (2016) estas características mejoran el rendimiento académico y Baumrind (1971), citado por Castillo (2016) señala que aumenta la confianza en sí mismos.

Se evidenció también que, aunque los padres se interesen por el avance académico de sus hijos, se siguen presentando prácticas de violencia al interior del sistema familiar. Según los resultados muchos padres no se basan frecuentemente en la razón para solucionar problemas, y no brindan herramientas con regularidad para que sus hijos solucionen los conflictos a los que se pueden enfrentar en los diversos contextos, resultados que concuerdan con los datos brindados por el Observatorio de Salud de Bogotá.

Con relación a la edad, los padres del tercer rango, no implementan algunas características como; brindar herramientas para solucionar conflictos, desarrollar la empatía, satisfacer las necesidades a partir de sus capacidades y entender que sus hijos piensen de manera autónoma, siendo la población que menos características implementa.

Los padres más jóvenes, aunque implementan todas las características, se evidencia que unas se emplean con mucha frecuencia y otras por el contrario son muy poco implementadas, como el uso de la razón en lugar del castigo físico. Situación contraria a los padres del segundo rango, ya que los resultados muestran tendencias muy estables de implementación de todas las características, debido a que los porcentajes no están por 
debajo del $17 \%$ ni por encima del 63\%. Resultados acordes con lo expuesto por La Rosa (1986), citado en Girardi y Velasco (2006) quien señala que los padres más jóvenes son más autoritarios, pero los padres mayores de 33 son más democráticos.

Frente al género, se evidenció que los padres identificados con género femenino se interesan por desarrollar la autonomía, independencia y formar y educar a los hijos. Hallazgos que se relacionan con lo expuesto por Carrillo et al. (2016) quienes afirman que partir de las transformaciones sociales las madres tratan de desarrollar en sus hijos e hijas las mismas habilidades.

Por otra parte, los padres de género masculino no implementan la razón para solucionar conflictos y desde sus capacidades no satisfacen las necesidades de sus hijos con regularidad. Resultados que se aproximan a lo expuesto por Carrillo et al. (2016) quien manifiesta que algunos hombres, siguen ejerciendo practicas hegemónicas y autoritarias en la crianza de sus hijos.

En cuanto a las limitaciones, se evidenció un desequilibrio en el número de participantes que tenían más de 60 años y en los padres que se identificaron con el género masculino.

Frente a la línea de investigación, los resultados permitieron identificar que los padres pueden emplear diferentes características de cada estilo. Se recomienda a futuros investigadores, estudiar la inclinación de los padres con relación a los tres estilos de crianza descritos, para determinar si algunos prevalecen en la población bogotana y ampliarlo a otras poblaciones. También se recomienda investigar si los hijos perciben el mismo estilo de crianza que creen los padres que emplean o implementan.

\section{CONCLUSIONES}

En conclusión, las características de la crianza democrática implementadas con mayor frecuencia por los padres residentes de la ciudad de Bogotá son; buscar la independencia y autonomía de los hijos, comunicarse de manera clara con ellos e interesarse por la formación y educación de los hijos.

También se puede concluir que las características menos implementadas son; brindar herramientas a los hijos para resolver problemas, satisfacer las necesidades afectivas, físicas y académicas de los hijos a partir de las capacidades que cada padre posee, y 
basarse en la razón y no en la imposición de la fuerza en la crianza de sus hijos.

Características que deben ser promovidas al interior del núcleo familiar.

Por otra parte, los padres de familia siguen empleando prácticas de violencia en lugar de usar la razón para solucionar los conflictos familiares, aunque se vivan transformaciones contundentes en cuanto a la equidad de género, la igualdad de oportunidades y la ruptura de estereotipos, los padres no consideran con regularidad, que los roles deben ser equitativos. Por lo anterior, se puede pensar que no se fomenta la igualdad de derechos con mucha frecuencia al interior de las familias.

Para finalizar, es importante resaltar el impacto que tiene la familia, en especial las prácticas de crianza que emplean los padres, en el desarrollo cognitivo, emocional y social de los niños, niñas y adolescentes. Por lo anterior, se considera fundamental promover el conocimiento y la puesta en práctica de un estilo de crianza más democrático y menos autoritario o permisivo.

\section{REFERENCIAS}

Acosta; Toro \& Guerrero (2015). Estilos parentales de enseñanza en familias con niños con necesidades educativas especiales en Samacá- Boyacá. Revista Praxis. Recuperado de http://revistas.unimagdalena.edu.co/index.php/praxis/article/view/1550/990

Arciniegas et al (2018). "Estilos parentales, función familiar y rendimiento académico: un estudio de caso de seis familias de un colegio público en Bogotá, Colombia". Voces y Silencios. Revista Latinoamericana de Educación 9(2) 4-36. Recuperado de: https://revistas.uniandes.edu.co/doi/full/10.18175/vys9.2.2018.02

Carrillo et al (2016). Percepciones del padre sobre su rol e involucramiento en la familia: un estudio cualitativo en una muestra colombiana. Revista Costarricense de Psicología, Jul-Dic 2016, 35(2) p. 101-118. Recuperado de: http://rcps-cr.org/?p=1964

Castillo (2016). Estilos de crianza de los padres de familia de los alumnos de $2^{\circ}$ grado de educación primaria de la I.E María Goretti de Castilla. Piura. Recuperado de https://pirhua.udep.edu.pe/bitstream/handle/11042/2564/EDUC_045.pdf?sequence= 1

Capano, González \& Massonnier. (2016). Estilos relacionales parentales: estudio con adolescentes y sus padres. Scielo. 34(2) ISSN 0254-9247 Recuperado de http://www.scielo.org.pe/pdf/psico/v34n2/a09v34n2.pdf

Colprensa (2020). Aprobada en la Cámara la ley que prohíbe castigo físico para castigar a los menores. Varnguardia. Recuperado de 
https://www.vanguardia.com/politica/aprobada-en-la-camara-la-ley-que-prohibecastigo-fisico-para-castigar-a-los-menores-El2468644

Echeverría, H.D. (2016). Diseños de investigación cuantitativa en psicología y educación. Ed. UniRío. Córdoba, Argentina. ISBN 978-987-688-166-1. Pág. 48-50

García, Rodríguez, Duarte \& Bermúdez-Jaimes (2017). Las prácticas de crianza y su relción con el vínculo afectivo. Revista Iberoamericana de psicología. Recuperado de https://reviberopsicologia.ibero.edu.co/article/view/970

Girardi y Velasco (2006). Padres autoritarioa y democráticos y características de personalidad de estudiantes de licenciatura y posgrado. Revista Intercontinental de $\begin{array}{lllll}\text { Psicología y } & \text { Educación (8) } 1 . & \text { Recuperado de }\end{array}$ https://www.redalyc.org/pdf/802/80280103.pdf

Guallpa \& Loja (2015). Estilos de crianza de los padres de estudiantes con bajo rendimiento académico. Tesis. Universidad de Cuenca. Recuperado de http://dspace.ucuenca.edu.ec/bitstream/123456789/21872/1/TESIS.pdf

Gutiérrez \& Ardila (2016) Revisión de literatura "incidencia de las pautas de crianza en conductas autolíticas en adolescentes". Universidad Cooperativa de Colombia, Bucaramanga, Colombia. Recuperado de: https://repository.ucc.edu.co/handle/20.500.12494/1617

Guzmán, Bastidas. \& Mendoza (2019). Estudio del rol de los padres de familia en la vida emocional de los hijos. Apuntes Universitarios, ISSN-e 2225-7136, Vol. 9, №. 2, 2019, págs. 61-72. Recuperado de: https://dialnet.unirioja.es/servlet/articulo?codigo $=7237417$

Jorge \& González (2017). Estilos de crianza parental: una revisión teórica. Informes Psicológicos, 17(2), pp. 39-66. Recuperado de: https://dialnet.unirioja.es/servlet/articulo?codigo=7044268

Hernández, Fernández \& Baptista. (2014). Metodología de la investigación. Sexta Edición. Editorial Mc Graw Hill. México. 2014• Hernández, R. Metodología de la Investigación. 6a Edición, Mc Graw Hill, México.

Infante \& Martínez (2016). Concepciones sobre la crianza: El pensamiento de madres y padres de familia. Revista Scielo. Recuperado de http://www.scielo.org.pe/scielo.php?pid=S1729$48272016000100003 \&$ script=sci_arttext\&tIng=en

Márquez \& Gaeta (2017). Desarrollo de competencias emocionales en preadolescentes: el papel de padres y docentes. Revista Electrónica Interuniversitaria de Formación del Profesorado, 20(2), 221-235.

Moreno (2013) Familias cambiantes, paternidad en crisis. Psicología desde el Caribe, vol. 30, núm. 1, enero-abril, 2013, pp. 177-209.Universidad del Norte. Barranquilla, Colombia. Recuperado de: https://www.redalyc.org/articulo.oa?id=21328600009 
Moreno, Ochoa \& Quintero (2017). Pautas y estilos de crianza presentes en las familias hondureñas en el año 2015. Revista Ciencia Y Tecnología, (20), 177-191. DOl: https://doi.org/10.5377/rct.v0i20.5502

Observatorio de Salud de Bogotá (2019). Tasa de Maltrato Infantil en Bogotá 2019. Recuperado de: https://saludata.saludcapital.gov.co/osb/index.php/datos-desalud/salud-mental/maltrato-infantil/

Parra y Rubio (2017). Una mirada desde el modelo ecológico de Bronfenbrenner de dos historias de sujetos que se convirtieron en padres/madres durante la adolescencia. Tesis de grado. Fundación Universitaria los Libertadores. Recuperado de https://repository.libertadores.edu.co/bitstream/handle/11371/1572/parrapaola2017. pdf? sequence $=1$ \&isAllowed $=y$

Rojas (2015). Felicidad y estilos de crianza parental. ESRU. Recuperado de https://ceey.org.mx/wp-content/uploads/2018/06/16-Rojas-2015.pdf 